\title{
SECURITY AND COLLISION IN RFID SYSTEMS
}

\author{
by \\ Mohammed Jameel Hakeem \\ MASc, University of Waterloo, Waterloo, Canada, 2008 \\ BASc, King Abdul-Aziz University, Jeddah, Saudi Arabia, 2004
}

\author{
A dissertation \\ Presented to Ryerson University \\ in partial fulfillment of the \\ requirements for the degree of \\ Doctor of Philosophy \\ in the Program of \\ Electrical and Computer Engineering
}

Toronto, Ontario, Canada, 2015

(C) Mohammed Jameel Hakeem 2015 


\section{SECURITY AND COLLISION IN RFID SYSTEMS}

by

Mohammed Jameel Hakeem MASc, University of Waterloo, Waterloo, Canada, 2008 BASc, King Abdul-Aziz University, Jeddah, Saudi Arabia, 2004

A dissertation

Presented to Ryerson University

in partial fulfillment of the

requirements for the degree of

Doctor of Philosophy

in the Program of

Electrical and Computer Engineering

Toronto, Ontario, Canada, 2015

CMohammed Jameel Hakeem 2015 


\section{Author's Declaration}

AUTHOR'S DECLARATION FOR ELECTRONIC SUBMISSION OF A DISSERTATION

I hereby declare that I am the sole author of this dissertation. This is a true copy of the dissertation, including any required final revisions, as accepted by my examiners.

I authorize Ryerson University to lend this dissertation to other institutions or individuals for the purpose of scholarly research

I further authorize Ryerson University to reproduce this dissertation by photocopying or by other means, in total or in part, at the request of other institutions or individuals for the purpose of scholarly research.

I understand that my dissertation may be made electronically available to the public. 


\section{DISSERTATION}

I hereby declare that I am the sole author of this dissertation. This is a true copy of the dissertation, including any required final revisions, as accepted by my examiners.

I authorize Ryerson University to lend this dissertation to other institutions or individuals for the purpose of scholarly research

I further authorize Ryerson University to reproduce this dissertation by photocopying or by other means, in total or in part, at the request of other institutions or individuals for the purpose of scholarly research.

I understand that my dissertation may be made electronically available to the public.

ii 
Security and Collision in RFID Systems, PhD, 2015, Mohammed Hakeem, Electrical \& Computer Engineering, Ryerson University.

\begin{abstract}
Radio Frequency Identification (RFID) is a promising technology to provide automated contactless identification of objects, people and animals. The identification process is performed as the reader receives simultaneous responses from various tags over a shared wireless channel and without no requirement of line-of-sight in the interrogation zone. The communication between the reader and tags is separated into two processes: identification and acknowledgment processes. Both processes suffer from serious drawbacks that limit the proliferation of RFID. Such drawbacks are security and privacy and collision problems.

This thesis has two main parts. The first part examines the security and privacy of the existing RFID authentication protocols. We introduced a novel cryptographic scheme, Hacker Proof Authentication Protocol (HPAP) that allows mutual authentication and achieves full security by deploying tag static identifier, updated timestamp, a one-way hash function and encryption keys with randomized update using Linear Feedback Shift Register (LFSR). Cryptanalysis and simulation show that the protocol is secure against various attacks. In comparison with the various existing RFID authentication protocols, our protocol has less computation load, requires less storage, and costs less.

The second part focuses on solving RFID collision arbitration imposed by the shared wireless link between a reader and the many tags distributed in the interrogation zone. In most proposed anticollision algorithms, tags reply randomly to the time slots chosen by the reader. Since more than two tags may choose the same time slot in a frame, this Random Access (RA) causes garbled data at the reader side resulting the identification process fails. Towards this challenge, two ALOHA based anti-collision algorithms that adopt a new way for tags to choose their replied time slots to enhance system efficiency are presented. In MBA and LTMBA, tags use modulo function to choose their owned time slot. The difference between the two algorithms relies on the method by which the reader estimates the next frame size. The performance evaluation of the two algorithms shows better performance than previously proposed algorithms in terms of fewer communication rounds and fewer collided/empty slots considering the limitation of the EPCglobal Class-1 Gen-2 standard.
\end{abstract}


Computer Engineering, Ryerson University.

Abstract Radio Frequency Identification (RFID) is a promising technology to provide automated contactless identification of objects, people and animals. The identification process is performed as the reader receives simultaneous responses from various tags over a shared wireless channel and without no requirement of line-of-sight in the interrogation zone. The communication between

the reader and tags is separated into two processes: identification and acknowledgment processes.

Both processes suffer from serious drawbacks that limit the proliferation of RFID. Such drawbacks

are security and privacy and collision problems.

This thesis has two main parts. The first part examines the security and privacy of the existing RFID authentication protocols. We introduced a novel cryptographic scheme, Hacker Proof Authentication Protocol (HPAP) that allows mutual authentication and achieves full security by deploying tag static identifier, updated timestamp, a one-way hash function and encryption keys with randomized update using Linear Feedback Shift Register (LFSR). Cryptanalysis and simulation show that the protocol is secure against various attacks. In comparison with the various

existing RFID authentication protocols, our protocol has less computation load, requires less storage, and costs less.

The second part focuses on solving RFID collision arbitration imposed by the shared wireless link

between a reader and the many tags distributed in the interrogation zone. In most proposed anticollision algorithms, tags reply randomly to the time slots chosen by the reader. Since more than two tags may choose the same time slot in a frame, this Random Access (RA) causes garbled data

at the reader side resulting the identification process fails. Towards this challenge, two ALOHA based anti-collision algorithms that adopt a new way for tags to choose their replied time slots to enhance system efficiency are presented. In MBA and LTMBA, tags use modulo function to choose their owned time slot. The difference between the two algorithms relies on the method by which the reader estimates the next frame size. The performance evaluation of the two 
algorithms

shows better performance than previously proposed algorithms in terms of fewer communication rounds and fewer collided/empty slots considering the limitation of the EPCglobal Class-1 Gen-2 standard.

iii 


\section{Acknowledgment}

First of all, I am grateful to God (Allah), The Most Beneficent, The Most Merciful. Him and only gave me and keeps giving me the strength to bring out the best of me to success in life.

It is difficult to overstate my gratitude to my Ph.D. supervisors, Dr. Kaamran Raahemifar and Dr.

Gul Khan. Their enthusiasm, inspiration, and great efforts to explain things clearly and simply, have helped me to better understand the field of security and optimization. Throughout my thesiswriting period, Dr. Kamran provided encouragement, sound advice, good teaching, good company, and many good ideas. I truly would have been lost without him. It was an honor and a privilege to work under the joint supervision of Dr. Kaamran Raahemifar and Dr. Gul Khan. In addition, I would like to extend my gratitude to my PhD defense committee; Chair - Dr. Paul Moore, External -Dr. Mustapha Yagoub, Dr. Alagan Anpalagan, Dr. Alireza Sadeghian, Dr. Nagi Mekhiel for their valuable comments in the internal and external defense.

My deepest gratitude extend to the Government of the Kingdom of Saudi Arabia, for the great chance to pursue my graduate studies through the custodian of the two holy mosques scholarship program and King Abdul-Aziz University. Many Thanks every and single supervisor I dealt with at the Saudi cultural Bureau in Ottawa. I specifically want to thank, Dr. Faisal Aba-Al-Khail, Dr. Ali Al-Bishri, Dr. Nasser Al-Shalaan, Dr. Amal Alamoudi, Dr. Samiah Al-Jabri and my supervisor Suha Mansour. Finally, I want to thank Dr. Majid Alotaibi, Dr. Danilo Yu, Eng. Haitham Turkistani for their help towards my research.

Certain people have giving me ultimate support and encouragement, having them in my live is a bless. Thank you to my beloved wife Maram Bajri; she was the best support to me every second. My great thanks to my all friends who never hesitated to give me valuable advises in life and school. Specifically, Dr. Omar Attas, Dr. Nezar Bahabri, Dr. Hassan Huwait, Dr. Abdullah Aldammak, Dr. Rami Kinsara and Enrique.

Lastly, I am extremely grateful to my family, Mom (Najiya), Essra, Bassem, Yasser, Hamza, battal and little cute Miral for their constant support and encouragement.

iv 
Merciful. Him and only

gave me and keeps giving me the strength to bring out the best of me to success in life.

It is difficult to overstate my gratitude to my Ph.D. supervisors, Dr. Kaamran Raahemifar and Dr.

Gul Khan. Their enthusiasm, inspiration, and great efforts to explain things clearly and simply, have helped me to better understand the field of security and optimization. Throughout my thesis-

writing period, Dr. Kamran provided encouragement, sound advice, good teaching, good company,

and many good ideas. I truly would have been lost without him. It was an honor and a privilege to

work under the joint supervision of Dr. Kaamran Raahemifar and Dr. Gul Khan. In addition, I would like to extend my gratitude to my PhD defense committee; Chair - Dr. Paul Moore, External

- Dr. Mustapha Yagoub, Dr. Alagan Anpalagan, Dr. Alireza Sadeghian, Dr. Nagi Mekhiel for their

valuable comments in the internal and external defense.

My deepest gratitude extend to the Government of the Kingdom of Saudi Arabia, for the great chance to pursue my graduate studies through the custodian of the two holy mosques scholarship program and King Abdul-Aziz University. Many Thanks every and single supervisor I dealt with at the Saudi cultural Bureau in Ottawa. I specifically want to thank, Dr. Faisal Aba-Al-Khail, Dr. Ali Al-Bishri, Dr. Nasser Al-Shalaan, Dr. Amal Alamoudi, Dr. Samiah Al-Jabri and my supervisor

Suha Mansour. Finally, I want to thank Dr. Majid Alotaibi, Dr. Danilo Yu, Eng. Haitham Turkistani for their help towards my research.

Certain people have giving me ultimate support and encouragement, having them in my live is a bless. Thank you to my beloved wife Maram Bajri; she was the best support to me every second. My great thanks to my all friends who never hesitated to give me valuable advises in life and school. Specifically, Dr. Omar Attas, Dr. Nezar Bahabri, Dr. Hassan Huwait, Dr. Abdullah Aldammak, Dr. Rami Kinsara and Enrique.

Lastly, I am extremely grateful to my family, Mom (Najiya), Essra, Bassem, Yasser, Hamza, battal 
and little cute Miral for their constant support and encouragement. iv 


\section{Dedication}

To

My Dad "Jameel" who never get to see this moment

I miss you baba - RIP 


\section{Dedication}

To

My Dad "Jameel" who never get to see this moment

I miss you baba - RIP

V 


\section{Table of Contents}

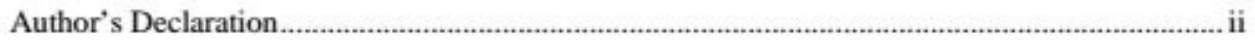

Abstract

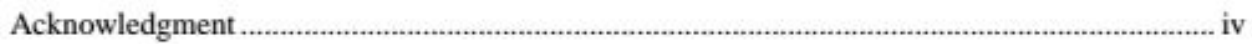

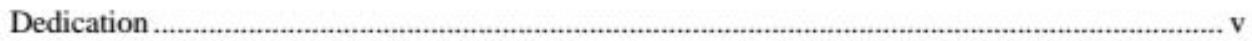

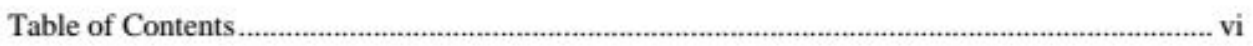

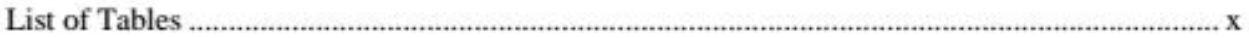

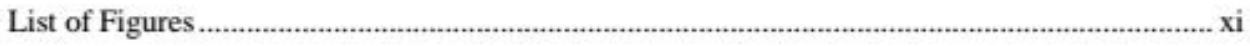

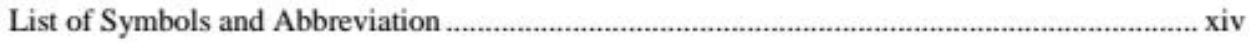

1. Chapter 1 .

Motivation and Contributions …..........................................................................................

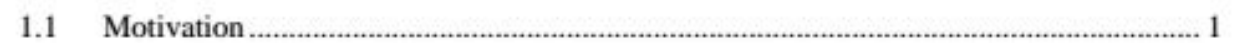

1.2 Objectives

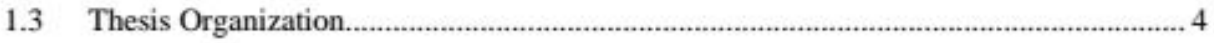

2. Chapter 2

Introduction to Radio Frequency Identification .............................................................. 6

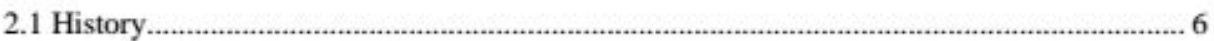

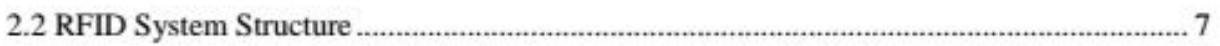

2.2 .1 RFID Tags

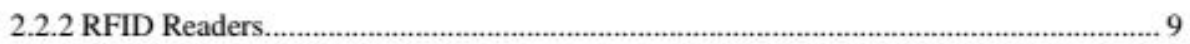

2.2.3 Back-end Server

2.3 RFID Standards

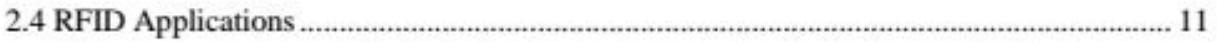

2.4.1 Supply chain management applications ........................................................... 11

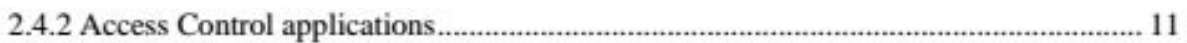


Abstract

iii

Acknowledgment

iv

Dedication

$\mathrm{V}$

Table of Contents

vi

List of Tables

$\mathrm{X}$

List of Figures

xi

List of Symbols and Abbreviation

xiv

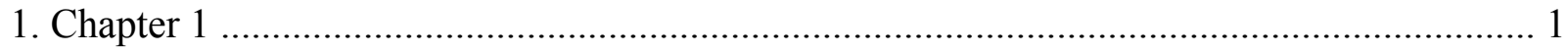

Motivation and Contributions

1

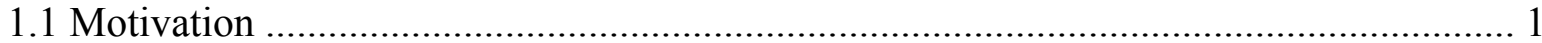

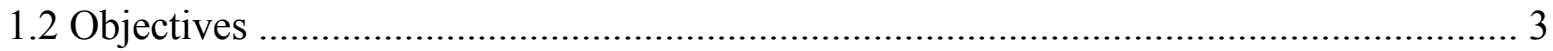

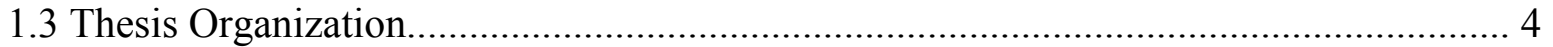

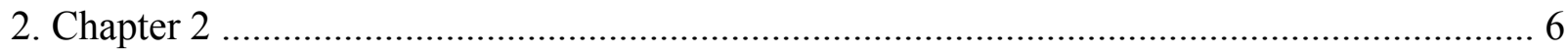

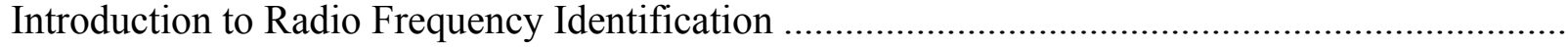

6

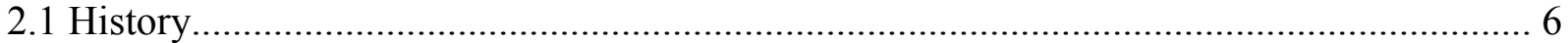

2.2 RFID System Structure ...................................................................................... 7

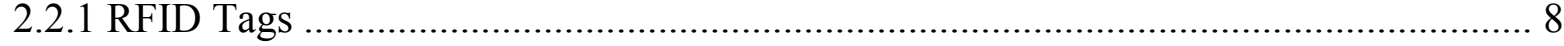

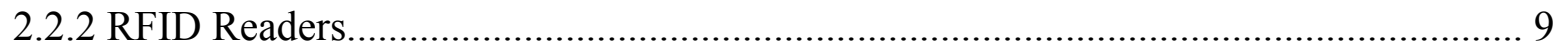

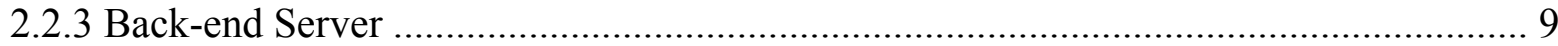

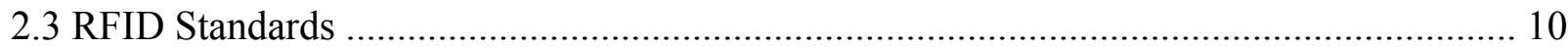

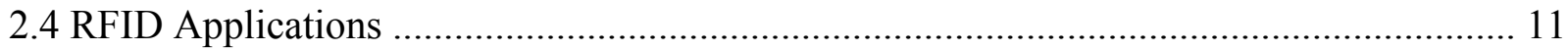


2.4.1 Supply chain management applications ………………............................................... 11

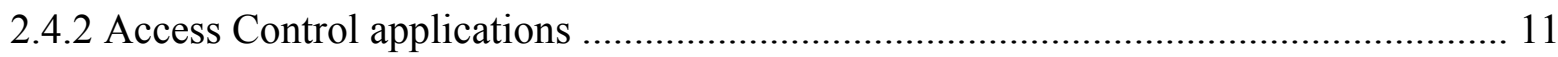
vi 
2.4.3 Cashless Payment Applications ......................................................................... 12

2.4.4 Animal and human tracking applications ........................................................... 13

2.4.5 Healthcare Industry Applications …......................................................................... 14

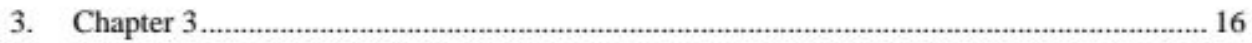

Privacy and Authentication in Radio Frequency Identification............................................ 16

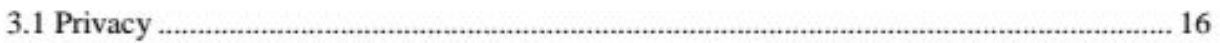

3.2 Privacy requirements ................................................................................................. 17

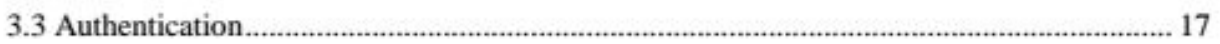

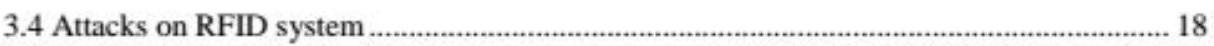

3.5 Providing Security Requirements ............................................................................. 19

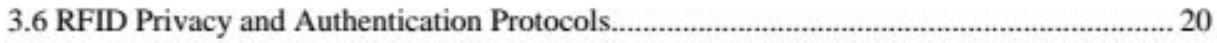

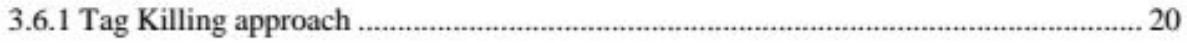

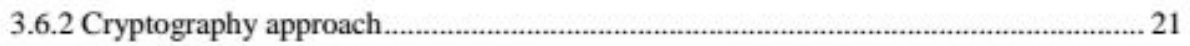

3.7 An Overview on RFID Authentication Protocols........................................................... 22

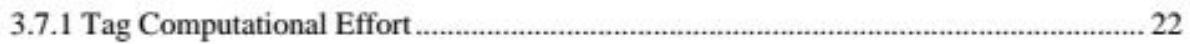

3.7.2 Tag Storage Capabilities ........................................................................................... 22

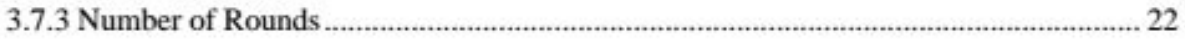

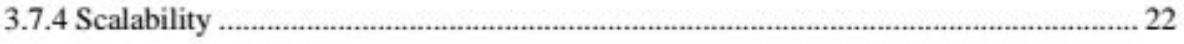

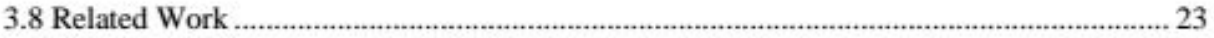

3.8.1 Hash Function Based Authentication Protocols ................................................... 23

3.8.2 Lightweight Based Authentication Protocols ........................................................ 27

3.8.3 Ultralightweight Authentication Protocol ................................................................. 36

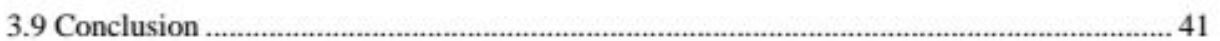

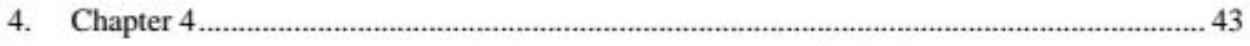

Collision in Radio Frequency Identification Systems ............................................................... 43

vii

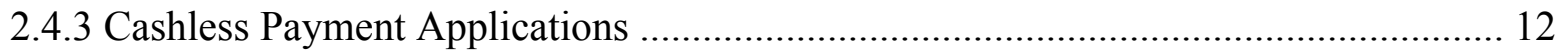




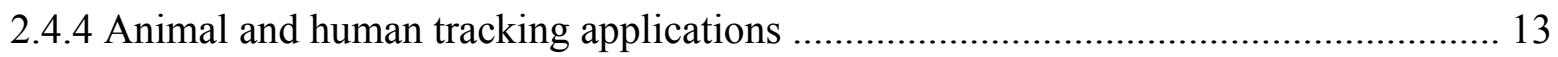

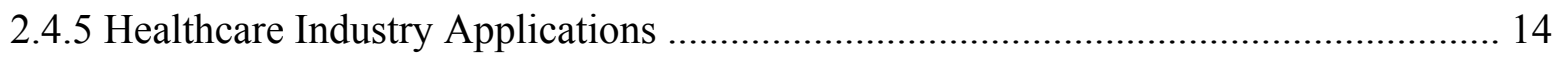

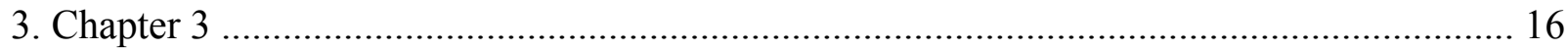

Privacy and Authentication in Radio Frequency Identification 16

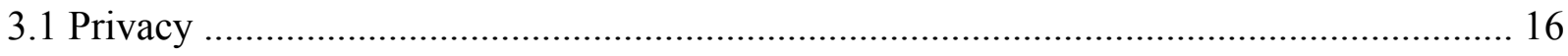

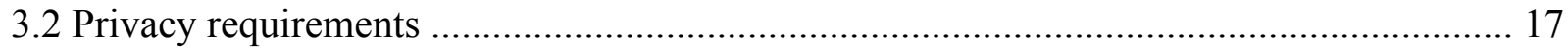

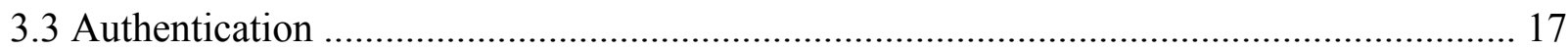

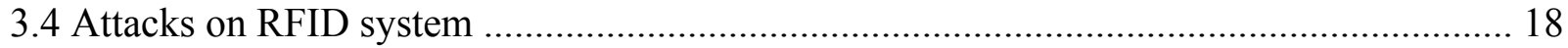

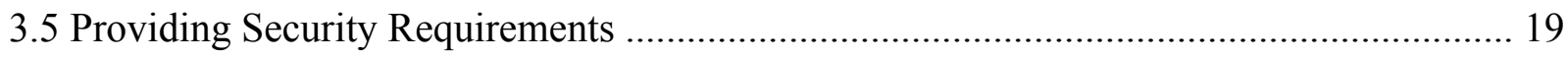

3.6 RFID Privacy and Authentication Protocols................................................................... 20

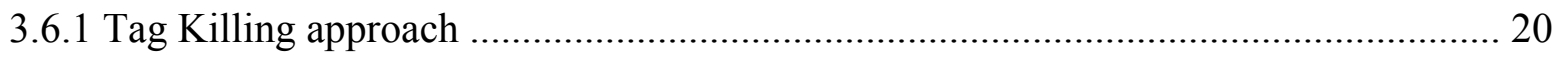

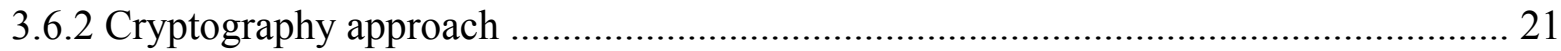

3.7 An Overview on RFID Authentication Protocols .......................................................... 22

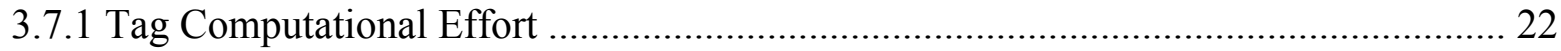

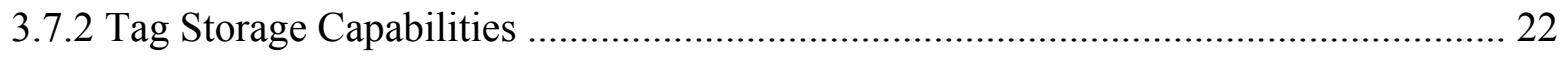

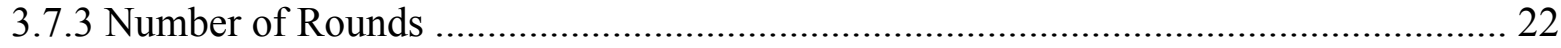

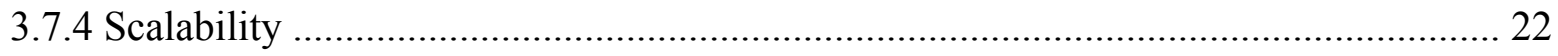

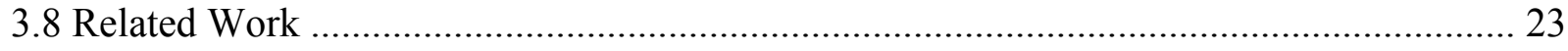

3.8.1 Hash Function Based Authentication Protocols ......................................................... 23

3.8.2 Lightweight Based Authentication Protocols ................................................................ 27

3.8.3 Ultralightweight Authentication Protocol ………....................................................... 36

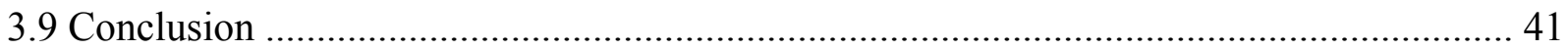

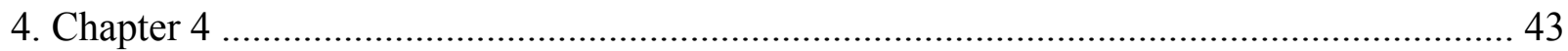

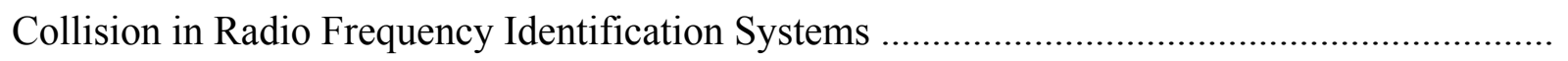
43

vii 


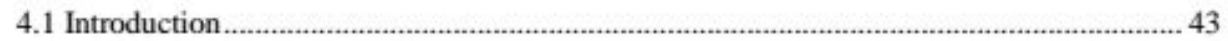

4.2 Estimation Techniques and Anti-Collision Protocols...................................................... 44

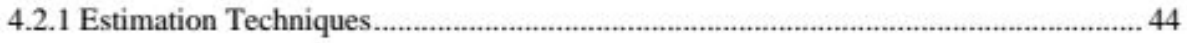

4.2.2 Tag Anti-Collision Algorithms ................................................................................. 48

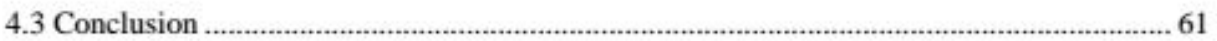

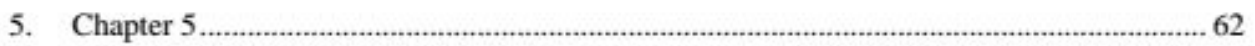

Key Management Protocol for Radio Frequency Identification Systems ................................... 62

5.1 Authentication Protocol Parameters ................................................................................ 62

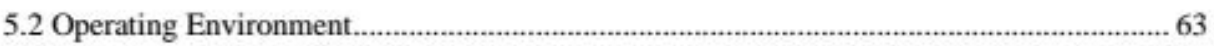

5.3 HPAP Component

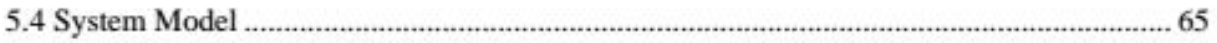

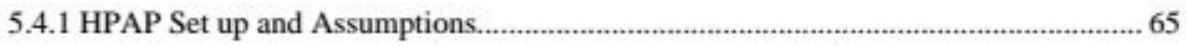

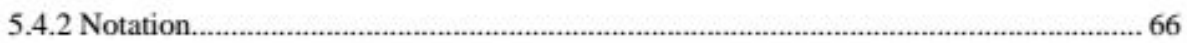

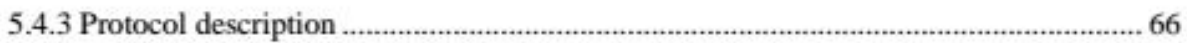

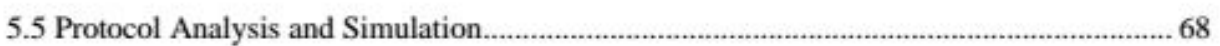

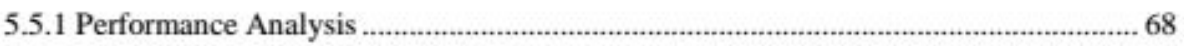

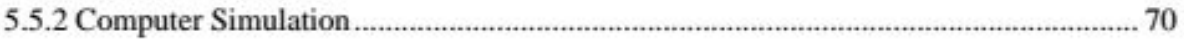

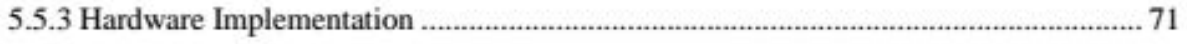

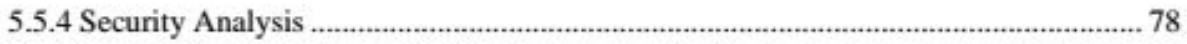

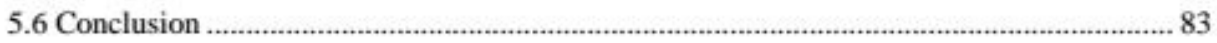

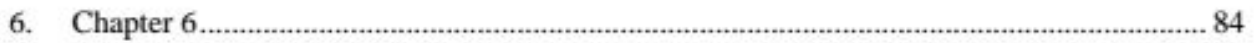

Modulo based Aloha Anti-Collision Algorithms for Radio Frequency Identification Systems... 84

6.1 EPC GLOBAL CLASS 1 GENERATION 2 STANDARD ........................................ 84

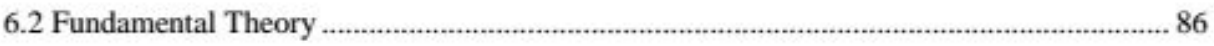

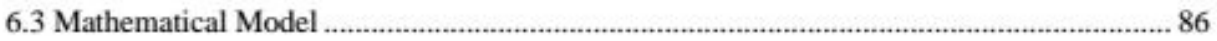

viii 
4.2 Estimation Techniques and Anti-Collision Protocols

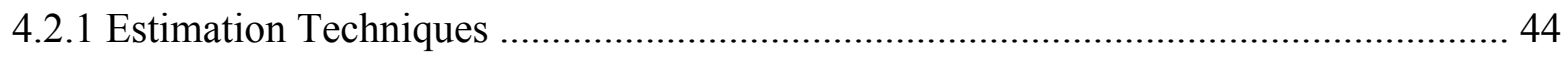

4.2.2 Tag Anti-Collision Algorithms ...................................................................... 48

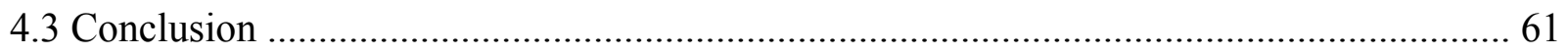

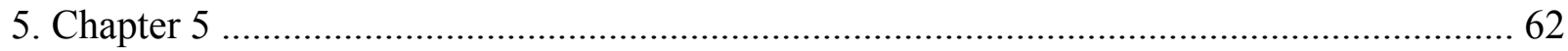

Key Management Protocol for Radio Frequency Identification Systems 62

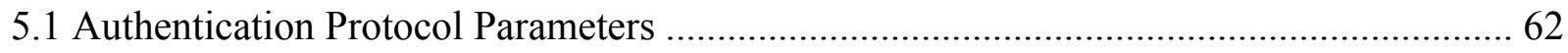

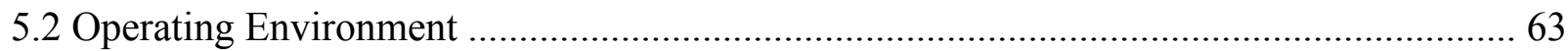

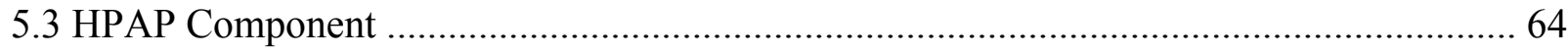

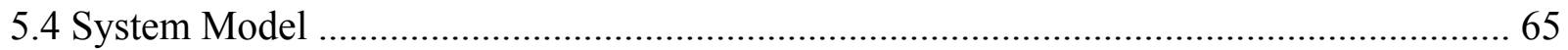

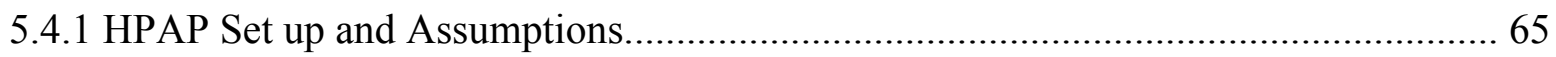

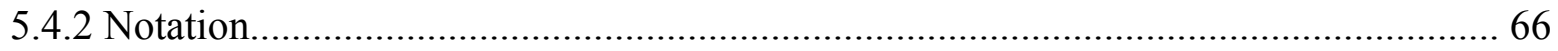

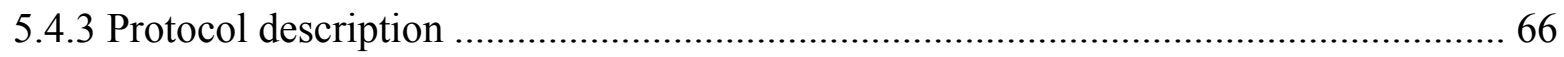

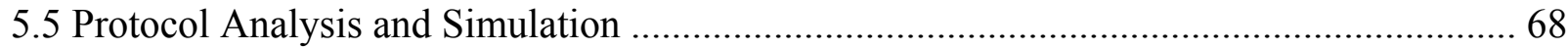

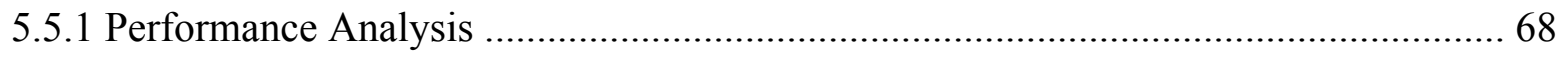

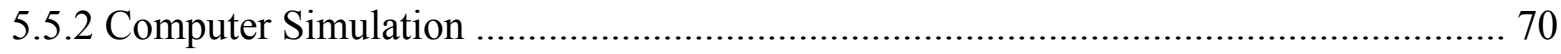

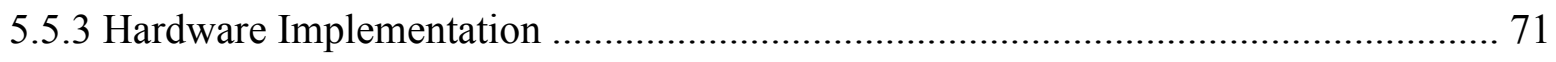

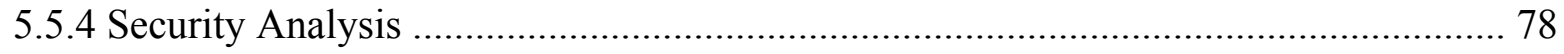

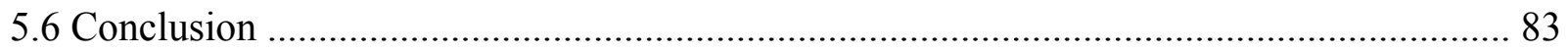

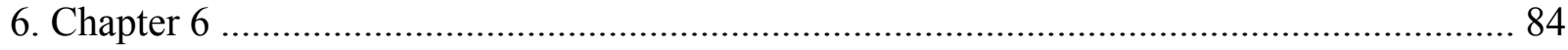

Modulo based Aloha Anti-Collision Algorithms for Radio Frequency Identification Systems ... 84

6.1 EPC GLOBAL CLASS 1 GENERATION 2 STANDARD ........................................ 84

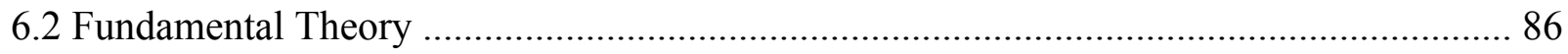

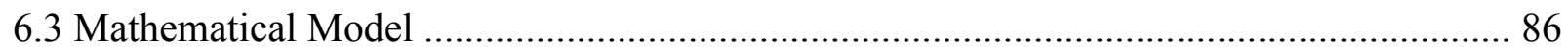

viii 


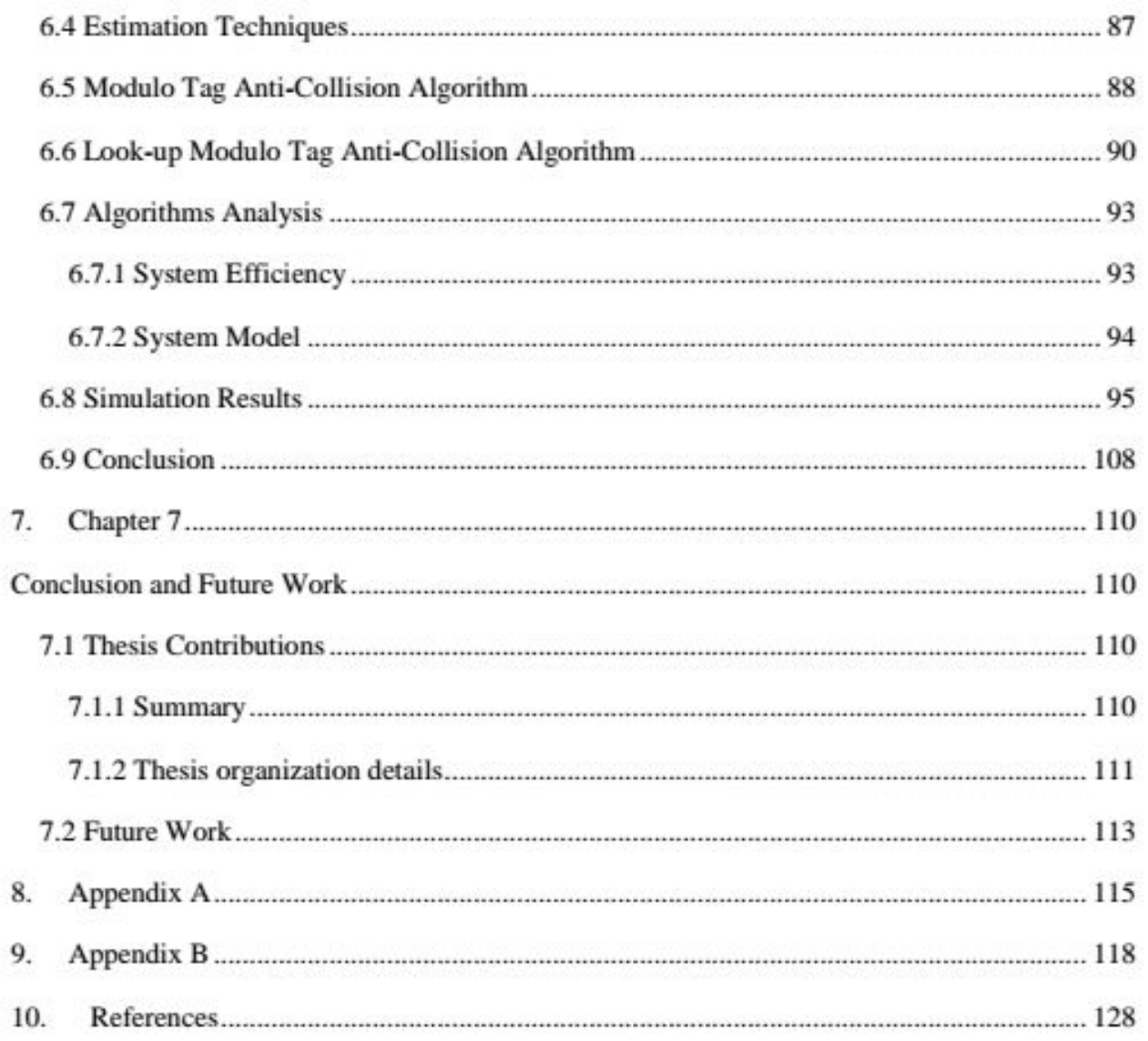

\section{ix}




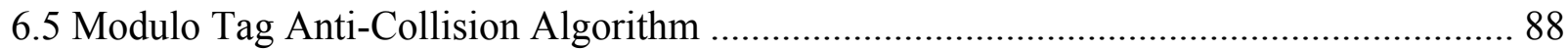

6.6 Look-up Modulo Tag Anti-Collision Algorithm ............................................................ 90

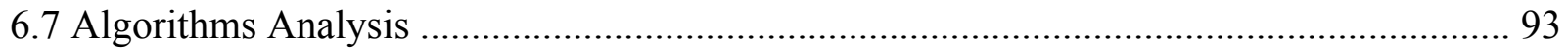

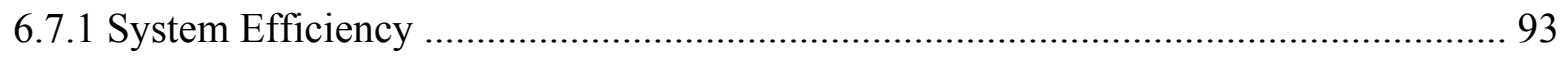

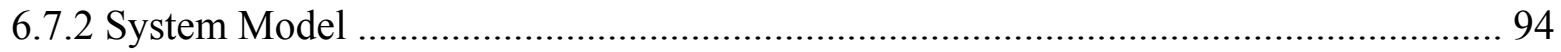

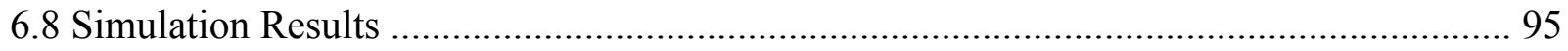

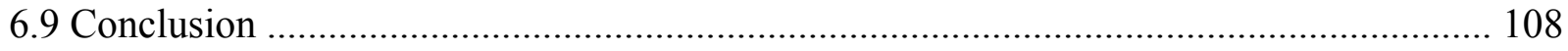

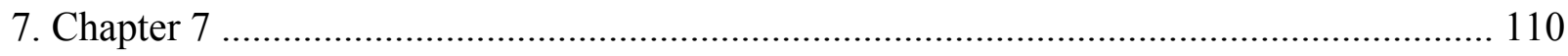

Conclusion and Future Work

110

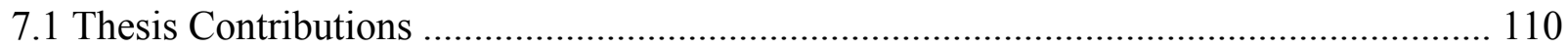

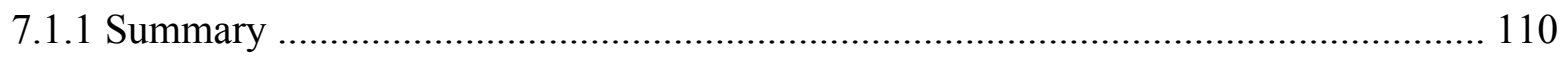

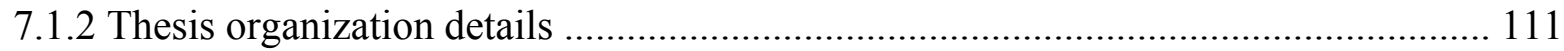

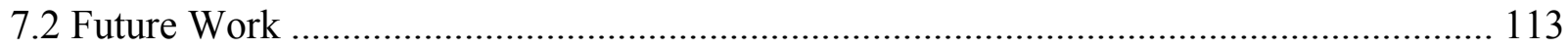

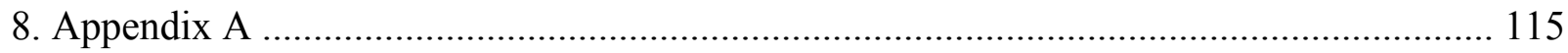

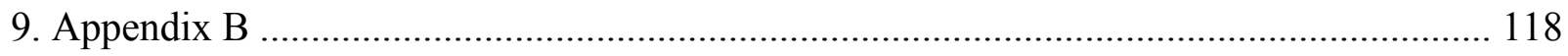

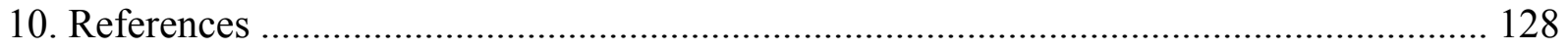

ix 


\section{List of Tables}

Table 4.1 Tag Anti-Collision Algorithms Comparison ............................................................ 59

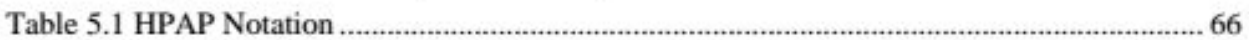

Table 5.2 Comparing the memory storage offered by HPAP and other authentication protocols 68 Table 5.3 Comparing the communication cost of HPAP with that of other authentication

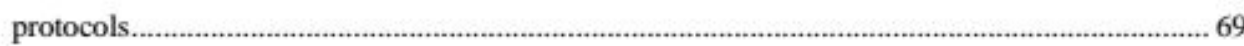

Table 5.4 Comparing the computational load of HPAP protocol with that of other authentication

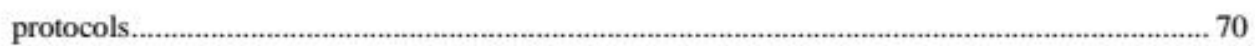

Table 5.5 Comparing the security offered by HPAP and other authentication protocols............. 83

Table 6.1 A Sample of the Pre-Saved Reader's Table............................................................ 92

Table 6.2 Proposed Anti-collision algorithms comparison; for 8 tags and initial NF=4 ............ 96

Table 6.3 Comparing different anti-collision algorithms; for 1000 tags and initial NF=32...... 103 
Table 5.1 HPAP Notation

66

Table 5.2 Comparing the memory storage offered by HPAP and other authentication protocols 68

Table 5.3 Comparing the communication cost of HPAP with that of other authentication protocols

69

Table 5.4 Comparing the computational load of HPAP protocol with that of other authentication protocols

70

Table 5.5 Comparing the security offered by HPAP and other authentication protocols 83

Table 6.1 A Sample of the Pre-Saved Reader's Table 92

Table 6.2 Proposed Anti-collision algorithms comparison; for 8 tags and initial NF=4 96

Table 6.3 Comparing different anti-collision algorithms; for 1000 tags and initial NF=32 ...... 103

$\mathrm{X}$ 